\title{
EL TRABAJO METODOLÓGICO MEDIADO POR EL ENFOQUE COMUNICATIVO EN LA UNIVERSIDAD DE ORIENTE: VALIDACIÓN DE UNA EXPERIENCIA RENOVADORA
}

\author{
Bárbara Fonseca Arias, Dra. C. \\ Universidad de Oriente, Cuba \\ barbaraf@uo.edu.cu
}

\author{
Elsa Iris Montenegro Moracén, Dra. C. \\ Universidad de Oriente, Cuba \\ elsam@uo.edu.cu
}

Palabras claves: trabajo metodológico colectivo, proceso formativo, eslabón de base, enfoque comunicativo.

Keywords: joint methodological work, educative process, academic year as the basis, communicative approach.
Recibido: 24 de Octubre de 2017

Aceptado: 17 de Noviembre de 2017

\section{RESUMEN}

La actual Universidad de Oriente es resultado del proceso de integración de tres instituciones de educación superior en Santiago de Cuba, lo cual condujo a replantearse las maneras de interactuar metodológicamente para incidir, a través de una labor coherente y pertinente, en la calidad del proceso formativo de pregrado en el eslabón de base: el enfoque comunicativo constituyó premisa esencial para su concreción. El presente trabajo tiene como objetivo mostrar una experiencia renovadora a través del trabajo metodológico colectivo implementado desde la Dirección de Formación del Profesional cuyos resultados tuvieron un efecto significativo en el proceso docente educativo desarrollado por el claustro universitario en las carreras.

\section{ABSTRACT}

The present Oriente University is the result of the integration process of three high education institutions in Santiago de Cuba, which enhances a new way of interacting from the methodological point of view. This coherent and suitable work contributes to achieve the quality in the education process of the future professionals centered in the academic year as the basis: the communicative approach was an essential premise in its concretion. The present paper aims at exposing an interesting experience related to the joint methodological work developed by the Professional Education Direction, which allowed some changes in the educative process carried out by the staff of the different majors. 


\section{INTRODUCCIÓN}

El trabajo metodológico en las universidades cubanas es una arista fundamental del quehacer profesional al constituirse en la vía para el perfeccionamiento sistemático de la labor docente; tal como precisa Cordoves-Mustelier (2014, p.290): en la nueva universidad cubana se reconceptualiza el trabajo metodológico como gestión pedagógica y didáctica del proceso de formación de profesionales, integrando las diferentes modalidades, y busca resolver la contradicción entre lo administrativo y lo pedagógico y el trabajo que se desarrolla en la sede central y centros universitarios municipales que le permita a la universidad, apoyándose en las leyes de la pedagogía, la didáctica y su carácter sistémico, trazar la estrategia a seguir por los distintos colectivos pedagógicos en dicho proceso. Así pues, en la Universidad de Oriente, el trabajo metodológico adquiere una dimensión estratégica como consecuencia de haberse producido la integración de tres instituciones de educación superior, lo cual planteó a la Dirección de Formación del Profesional (DFP), como parte esencial de la gestión educativa institucional, la capacidad de generar procesos de transformación de la realidad mediante la participación colectiva en el diseño, decisión y evaluación del funcionamiento de los procesos.

Por tanto, una dinámica encaminada al perfeccionamiento del quehacer metodológico como vía para hacer más efectiva la labor educativa desde la instrucción en el proceso docente educativo que se concreta en la comunidad universitaria del eslabón de base que lo componen los departamentos docentes, el colectivo de carrera y la comunidad universitaria del año académico, representado por el profesor principal del año académico, los profesores guías, los tutores, el claustro de profesores y los estudiantes (Ministerio de Educación Superior, 2013). En ese eslabón de base, pues, desempeñan un papel fundamental los profesores y estudiantes; en la Universidad de Oriente la composición de su claustro es la siguiente: el 52,9 \% posee categoría docente superior de Profesor Titular o Auxiliar, el 86,4 $\%$ ostenta el grado de doctor $(24,6 \%)$ o el título de máster y especialista $(61,8 \%)$, mientras que los estudiantes conforman una elevada matrícula en tres modalidades de estudio: presencial (curso diurno), semipresencial (curso por encuentro), no presencial (curso a distancia). Vid a continuación:

\begin{tabular}{|l|l|l|l||}
\hline \hline Tipo de curso 2015-2016 2016-2017 & 2017-2018 \\
\hline Diurno & 6971 & 6660 & 6274 \\
\hline Por Encuentro & 2000 & 7181 & 7900 \\
\hline A Distancia & 222 & 513 & 457 \\
\hline UO & 9193 & 14354 & 14631 \\
\hline \hline
\end{tabular}

Lograr un trabajo metodológico colectivo, coherente y pertinente en la Universidad de Oriente, significó diseñarlo y organizarlo sobre la base de las mejores experiencias devenidas de los tres centros formadores integrados, en pos de favorecer el proceso formativo de pregrado, y en ese propósito el enfoque comunicativo, asumido bajo los postulados del proceso comunicativo, constituyó premisa fundamental desde la gestión y su 
proyección en los distintos niveles para responder a los principales problemas de la universidad, las facultades, centros universitarios municipales y departamentos independientes, teniendo como objetivo general el perfeccionamiento de la formación integral del educando, a fin de garantizar la calidad en el cumplimiento de los objetivos expresados en el modelo del profesional.

La finalidad del trabajo metodológico colectivo se asume, bajo una concepción de sistema, como proceso encaminado a propiciar, progresivamente, transformaciones en los modos de pensar y hacer por docentes y estudiantes, acordes con las aspiraciones de la formación de un profesional integral y con compromiso social, como demanda el modelo de formación. Para cumplir ese propósito, la comunicación que logró establecerse entre los diferentes agentes que intervienen en el proceso fue elemento cardinal que favoreció el tránsito por diferentes etapas: diagnóstico profundo de las limitaciones y potencialidades del trabajo metodológico en las universidades que se integraron; planificación actual y proyectiva; control, seguimiento y evaluación al desarrollo de las acciones metodológicas planificadas hasta el eslabón de base y la calidad del proceso formativo en general.

Dicho proceso se caracteriza por ser dialéctico, continuo, sistémico y sistemático, lo cual permite el replanteamiento de su concepción en cada una de esas etapas, guiadas por el quehacer del colectivo, para alcanzar la calidad del proceso formativo de pregrado, caracterizado por su carácter humanista, universalizado, científico, tecnológico e innovador. Este artículo centra su interés en los resultados de la validación de la experiencia que sobre el trabajo metodológico colectivo fue implementada como parte de la gestión educativa institucional por la Dirección de Formación del Profesional en la Universidad de Oriente, para cuyo desarrollo el enfoque comunicativo fue esencial.

\section{DESARROLLO}

\section{Fundamentos del trabajo metodológico colectivo en la Universidad de Oriente}

El Reglamento Docente y Metodológico de la educación superior cubana (Ministerio de Educación Superior, 2007) define en su artículo 24 que el trabajo metodológico es "[...] la labor que, apoyados en la Didáctica, realizan los sujetos que intervienen en el proceso docente educativo, con el propósito de alcanzar óptimos resultados en dicho proceso, jerarquizando la labor educativa desde la instrucción, para satisfacer plenamente los objetivos formulados en los planes de estudio" (p.14).

El trabajo metodológico en la formación profesional es la gestión de la didáctica, la cual es inherente a todos los procesos que tienen lugar en la educación superior y que supone planificar, organizar, regular y controlar el proceso formativo. Particularmente en el proceso docente educativo, es la actividad metodológica la que singulariza el sistema de trabajo del claustro dirigido a perfeccionarlo, optimizarlo, en correspondencia con las necesidades y exigencias de la formación de los profesionales de nivel superior, para poder materializar cabalmente los objetivos de los planes de estudio. 
Vale significar que el modelo de formación de la educación superior cubana, presenta particularidades para cada modalidad de estudio, es de perfil amplio y se sustenta en dos ideas rectoras fundamentales: la unidad entre la educación y la instrucción, que expresa la necesidad de educar al hombre a la vez que se instruye; y el vínculo del estudio con el trabajo, que consiste en asegurar desde el currículo el dominio de los modos de actuación del profesional, en vínculo directo con su actividad profesional ( Op.cit, p.9). Para su realización puede adoptar dos formas: individual y colectiva, esta última tiene como rasgo esencial el enfoque en sistema y se lleva a cabo en cada uno de los niveles organizativos del proceso docente educativo (carrera, año, disciplina, asignatura) pero también en los niveles de dirección: universidad, facultad, centro universitario municipal y departamento docente.

Sin embargo, lograr una concepción coherente del trabajo metodológico colectivo como sistema y su materialización desde la gestión educativa institucional conllevó a hacer replanteamientos, a la luz del contexto de la integración institucional, como parte del perfeccionamiento de los centros cubanos de educación superior, para incidir con mayor intencionalidad en la labor que realizan los profesores que integran el colectivo de la carrera y que mediante el trabajo coordinado y sistemático permite orientar, desde el punto de vista didáctico, el desarrollo de las disciplinas y los años en que se estructura la carrera, a fin de cumplir con el modelo del profesional, tomando como elemento fundamental el enfoque integral para la labor educativa. El colectivo metodológico de la Dirección de Formación del Profesional asume el trabajo metodológico colectivo como sistema y proceso en el sentido de que, mediado por un enfoque comunicativo, permita cumplir con coherencia y pertinencia las diferentes acciones que se definen por la dirección y que deben impactar en la calidad del proceso docente educativo que se concreta en el eslabón de base, escenario educativo en el cual la acción de los agentes que intervienen es determinante para lograr la formación integral del profesional, lo que significa, como refiere P. Horruitiner $(2009$, p.52), un profesional "[...] completamente preparado para desempeñarse en el eslabón de base de su profesión", toda vez que haya egresado.

Esa asunción ha estado encaminada a propiciar, progresivamente, transformaciones que se expresen en los modos de actuación de los docentes quienes tienen el encargo directo de educar a través de la instrucción y materializar su labor con un enfoque educativo, de conjunto con toda la comunidad universitaria en los diferentes escenarios de la formación. Para cumplir con ese propósito ha sido decisiva la comunicación. Es, pues, la comunicación, premisa que sustenta el trabajo metodológico colectivo, asociada a la relación entre los hombres; su significación depende de los sujetos implicados en ella y, al propio tiempo, las características de los sujetos determinan el proceso de comunicación.

Se considera una categoría polisemántica en tanto su utilización no es exclusiva de una ciencia social en particular, teniendo connotaciones propias de la ciencia social de que se trate; no obstante vale significar que como proceso social ha sido objeto de estudio particularmente de la Lingüística, la Psicología y la Sociología, ciencias que aportaron nuevas concepciones a partir de una comprensión más profunda de su importancia en todas las ramas del quehacer humano, y que han servido de apoyo a la Teoría de la Comunicación, la cual se erige sobre las categorías comunicación (conjunto del proceso comunicativo mediante el lenguaje), información (contenido de la comunicación) y aprendizaje (cuando las consecuencias del mensaje dotan al sujeto de ciertos principios, normas de conducta, 
criterios de valor, convicciones morales, guías de acción adquiridas no por manipulación, sino por conocimiento propio). (Abello Cruz et al, 2014, capítulo 1, p. 2).

Se asume con lleana Domínguez (2007, p.4) la comunicación desde una perspectiva humanista al colocar al hombre en el centro de las relaciones que establece con los demás sujetos y con el propio mundo, y como resultado de toda su actividad la cual permite conocer qué se ha hecho, cómo y para qué; esto es, permite transmitir todo el quehacer de una generación a otra e identificar su pertenencia a una clase social, a un grupo, desde una posición ideológica bien definida. Así pues, esos presupuestos como premisas del trabajo metodológico colectivo desde la gestión educativa institucional que desarrolla la Dirección de Formación del Profesional, hace que lo interpretemos como un proceso dinámico entre individuos y/o grupos que, mediante el intercambio informativo, sirve para establecer la comprensión dialéctica continua, sistémica y sistemática, desde la comunicación como forma de relación entre los individuos, que incluye lo científico, investigativo y extensionista, propone herramientas al eslabón de base de la formación en la búsqueda de soluciones a problemas metodológicos, tanto por la vía del trabajo docente como científico, identificados en los niveles organizativos y de dirección.

Tiene como fundamento el principio de derivación gradual que no significa la repetición exacta de lo que se planifica a nivel de universidad, sino que más bien posibilita adecuar el trabajo metodológico que se realiza en la base a partir de las líneas y objetivos generales que han sido definidos para su tratamiento (Fonseca et al, 2016). Requerimientos para el logro del trabajo metodológico colectivo, mediado por el enfoque comunicativo:

1) Seleccionar los agentes participantes en la variante colectiva de trabajo metodológico; para la cual se requiere de:

1.1. Conocer las competencias, compromiso y motivaciones profesionales de cada uno.

1.2. Tener en cuenta la preparación en aristas claves para el desarrollo del trabajo metodológico.

1.3. Considerar los tipos de inteligencia predominantes, su diversidad para, desde la estimulación de la interacción, potenciar la calidad en la ejecución de los procesos (dentro de ellas: inteligencia interpersonal, inteligencia intrapersonal, inteligencia lingüística, inteligencia lógico-matemática, inteligencia espacial (H. Gardner, 2008).

2) Diseñar y organizar la actividad del colectivo desde las potencialidades individuales, de manera que se realice actividad individual, para luego ser socializada, consensuada y concluir su realización desde la revisión y aprobación colectiva, lo que redundará en la calidad de las ejecuciones.

3) El desarrollo del trabajo metodológico colectivo, requiere de responsabilidad, modestia, profesionalidad colectiva, del accionar despojado de prejuicios al interior del colectivo; donde prime la confianza y camaradería, de modo que siempre se valore el resultado como colectivo y no desde la individualidad.

4) El establecimiento de principios y reglas básicas, como sustentos importantes para el trabajo metodológico colectivo. Dentro de los primeros deben considerarse: principios éticos, pedagógicos, el de derivación gradual, de descentralización, de las teorías de la 
información y la comunicación. Dentro de las reglas, se encuentran las básicas de la comunicación, en las que desempeñan un papel importante sus funciones esenciales: informativa (transmisión de conceptos, datos, información en general, se materializa mediante la función referencial del lenguaje), afectivo valorativa (transmisión de sentimientos y valoraciones, relacionada con la ética, creencias, la moral; se manifiesta a través de la función expresiva del lenguaje) y reguladora (establece la retroalimentación que hay en todo proceso comunicativo tanto para que el emisor pueda ver el efecto de su mensaje como para que el receptor se evalúe a partir de lo que le aporta la información recibida, es reguladora de conducta por el efecto en el receptor, y se manifiesta en la función conativa del lenguaje) (Domínguez, 2007, pp.23 y 24).

5) Adecuada planificación y control de las tareas a desarrollar, para que puedan ser valoradas en el colectivo, que haya participación y protagonismo de todos los miembros, para lograr la calidad en el cumplimiento de aquellas.

6) Los resultados del trabajo metodológico colectivo son expresión de las relaciones que median entre los participantes de la actividad metodológica común.

7) Determinación de etapas, concebidas para la organización, planificación, control del trabajo metodológico; y evaluación de resultados a partir de su implementación durante dos cursos académicos (2015-2016 y 2016-2017).

\section{Etapa 1. Diagnóstico de limitaciones y potencialidades del trabajo metodológico colectivo}

El diagnóstico de limitaciones y potencialidades se realizó a partir del estudio y análisis de los instrumentos que abajo se precisan:

- Documentación con la proyección del sistema de trabajo metodológico en facultades, carreras y centros universitarios municipales.

- Los informes de resultados del proceso docente educativo, planes de estudio y trabajo metodológico de facultades y centros universitarios municipales en el curso académico precedente de los centros formadores.

- Estrategias curriculares, para definir cuáles debían implementarse en la nueva universidad integrada, de acuerdo con las propuestas realizadas por cada Comisión Nacional de carrera, según el modelo del profesional y el perfil particular de la formación. Se significa que las Comisiones Nacionales de las carreras son las encargadas de dirigir, a nivel nacional, los aspectos esenciales del trabajo metodológico en cada carrera para asegurar la mejora continua de la calidad del proceso de formación. Está constituida por profesores con elevada experiencia docente y nivel científico reconocidos, así como por profesionales de la producción y los servicios con prestigio en su esfera laboral, y representantes de las organizaciones estudiantiles. La sede de cada Comisión Nacional de la carrera se denomina Centro Rector y se designa por el organismo correspondiente de la administración central del Estado, al centro de educación superior con mayor experiencia en el desarrollo de la carrera (MES, 2007, artículos 60 y 62, p. 27).

- Introducción de resultados científicos en el proceso formativo de las carreras a partir de los proyectos de investigación que se desarrollan. 
En el estudio y análisis de esos instrumentos participan de manera activa los asesores de la Dirección de Formación del Profesional y profesores de elevada calificación y experiencia integrados en un grupo asesor de esta dirección. En el caso particular del análisis de la proyección de trabajo metodológico ese grupo se encarga de hacer oponencias a los planes de trabajo metodológico los cuales, en las jornadas que se planifican al iniciase el curso académico, se presentan por los vicedecanos docentes y coordinadores de los colectivos de carrera (figuras que inciden en la conducción del trabajo metodológico en la comunidad universitaria del eslabón de base).

Este ejercicio permite delimitar las principales debilidades que fueron comunes, y que constituyeron regularidades, a partir de las cuales se pudo proyectar entonces, desde una concepción sistémica, la planificación del trabajo metodológico en la universidad y su contextualización en las 63 carreras que abarcan las ciencias naturales, exactas, económicas, técnicas, agropecuarias, jurídicas, sociales, humanísticas, pedagógicas, y de la cultura física, las cuales están organizadas en 13 facultades que radican en la sede central, de ellas, 50 se desarrollan en el curso diurno, 45 en el curso por encuentro y 14 tienen su sede en ocho centros universitarios municipales. En consecuencia, esa proyección considera los resultados del proceso docente educativo en el curso académico anterior, por considerarse expresión objetiva de la efectividad o no del trabajo metodológico; lo cual posibilita validar las mejores soluciones al tratamiento de los problemas metodológicos que han sido identificados por los niveles organizativos y de dirección, lo cual es supuesto esencial para asegurar la calidad del proceso de formación del profesional desde la gestión institucional. Así se llegan a determinar las debilidades que debían atenderse.

En el curso 2015-2016 estas estuvieron relacionadas con:

1) La concepción y control de los planes de trabajo metodológico en los diferentes niveles del proceso, fundamentalmente en el diagnóstico de las causas que inciden en los resultados docentes para la determinación de los problemas metodológicos a tratar en cada colectivo; la instrumentación de la educación a través de la instrucción desde el incremento de actividades metodológicas en los colectivos de carreras, disciplinas, asignaturas y años académicos que permitan explotar las potencialidades educativas de los contenidos de las asignaturas y la atención especial al tratamiento metodológico de las estrategias curriculares.

2) La preparación de los coordinadores de los colectivos de las carreras, jefes de departamentos, profesores principales de año académico y disciplina para garantizar el conocimiento profundo y la instrumentación de acciones que permitan el adecuado cumplimiento de sus funciones en el eslabón de base, de modo que se contribuya a elevar a planos superiores la formación integral de los estudiantes.

3) El control por los departamentos docentes, en particular de los colectivos de disciplinas y de año académico, a las acciones que realizan los profesores dirigidas a propiciar el incremento del estudio sistemático de los estudiantes y su consecuente efecto en la promoción y su calidad.

4) La adecuada correspondencia del trabajo docente y científico metodológicos de los departamentos con las necesidades pedagógicas de los colectivos, los resultados del 
diagnóstico y del proceso de enseñanza - aprendizaje de las disciplinas y asignaturas, así como de los controles a clases, que demuestre la concepción de las actividades que se realizan, su derivación en sistema y la retroalimentación, mediante el control.

5) La sistematización del trabajo científico metodológico, particularmente en los departamentos docentes, a fin de lograr la introducción de resultados de las investigaciones pedagógicas y no pedagógicas para el perfeccionamiento del proceso docente educativo.

Por su parte, el diagnóstico de limitaciones y potencialidades permitió identificar las debilidades que debían atenderse mediante el sistema de trabajo metodológico colectivo durante el curso 2016-2017.

a) Insuficiencias en la gestión desde el colectivo de carrera para contribuir con enfoque sistémico al cumplimiento con calidad del modelo del profesional, mediante la coordinación del trabajo metodológico de las disciplinas y los años.

b) Insuficiencias en la gestión de los departamentos docentes en la planificación y seguimiento al trabajo metodológico.

c) Insuficiencias en el control e instrumentación, mediante el trabajo metodológico, a la introducción de los resultados de las investigaciones (pedagógicas y no pedagógicas) en el proceso de formación del profesional.

d) Limitaciones en la coherencia del trabajo metodológico hacia los centros universitarios. municipales desde la sede central, con énfasis en la atención diferenciada a las singularidades didáctico-metodológicas de la modalidad de estudio semipresencial en el curso por encuentro.

e) Limitaciones en la atención desde el sistema de trabajo metodológico de los diferentes niveles organizativos y de dirección a las debilidades detectadas en los informes de evaluación externa de carreras realizada por la Junta de Acreditación Nacional, encargada del desarrollo y aplicación del sistema universitario de programas de acreditación (SUPRA) para los procesos que en las instituciones de educación superior cubana se desarrollan y tiene como objetivo fundamental promover la mejora continua (Junta de Acreditación Nacional, 2015, p. 7).

Otro instrumento analizado en la etapa de diagnóstico fue las estrategias curriculares. El concepto de estrategias curriculares, denominado también por algunos autores ejes transversales, expresa una cualidad necesaria al concebir el plan de estudio de una carrera universitaria, y está relacionado con aquellos objetivos generales que no son posibles alcanzarlos, con el nivel de profundidad y dominio requeridos, desde el contenido de una sola disciplina y demandan el concurso adicional de las restantes.

Se trata de una idea integradora, a nivel de todo el currículo, que va precisando año a año cuáles son las acciones a cumplir por cada disciplina, para al final de los estudios lograr un estudiante profesionalmente apto en el empleo de esas herramientas. Si la estrategia no se diseña adecuadamente, dejando en manos de cada uno de los profesores decidir cómo utilizan estos recursos, entonces el enfoque no tendrá la debida coherencia. 
En el modelo de formación cubano, tales acciones pasan a formar parte de los objetivos de cada uno de los años y con ello se asegura su adecuada gestión pedagógica. (Horruitiner, 2009, p. 44). Así pues, se realizó un estudio y análisis minucioso del contenido de las estrategias curriculares instrumentadas en los centros universitarios formadores de procedencia; posteriormente en relación con las aprobadas por las Comisiones Nacionales de carrera, se decidió cuáles se asumirían por las carreras, sometiéndose al estudio por parte de especialistas propuestos y designados para elaborar y conformar cada estrategia curricular, a partir de las orientaciones ofrecidas por la Dirección de Formación del Profesional, que aseguraron la homogeneidad estructural de estas, bajo un proceso de asesoramiento, intercambio y seguimiento, para su posterior contextualización e implementación.

Por su parte, el análisis del trabajo científico metodológico conllevó a realizar un levantamiento de los resultados que los proyectos de investigación aportaron a la formación de los profesionales de pregrado; en este sentido, también se incidió en la concepción de la planificación del trabajo metodológico en las facultades, departamentos docentes y centros universitarios municipales, con el propósito de que se potenciara la introducción de resultados investigativos en el proceso docente educativo y su socialización a través de los seminarios científicos metodológicos y la Conferencia Científica Metodológica de la universidad (CONCIMET 2016 y 2017).

El seguimiento sistemático desde la Dirección de Formación del Profesional hasta el eslabón de base impactó positivamente al registrarse en 2016, 776 resultados investigativos introducidos en la formación del profesional de pregrado, que contribuyeron a la actualización de materiales docentes, la conformación y uso de plataformas interactivas para la gestión del aprendizaje y el diseño curricular de los planes y programas de estudio, así como las concepciones didácticas y para la dirección de la labor educativa; todo lo cual posibilitó que en ambas ediciones de CONCIMET, se socializaron 120 y 189 resultados investigativos y/o experiencias pedagógicas respectivamente, que giraron en torno a las temáticas siguientes: Experiencias científico pedagógicas en la formación inicial y permanente de profesionales (Formación continua y Didáctica).

- La labor educativa, educación en valores. Formación integral y desarrolladora del ciudadano.

- Tecnologías de la información y las comunicaciones (TIC) en la formación profesional.

- La formación laboral e investigativa. Relaciones universidad - sociedad en la formación profesional.

- La orientación vocacional y reafirmación profesional en las carreras universitarias.

- La calidad en la formación del profesional. Evaluación y acreditación de carreras. El perfeccionamiento del currículo en la formación de profesionales.

\section{Etapa 2. Planificación proyectiva del trabajo metodológico}

La planificación quedó esbozada en los planes de trabajo metodológico de la universidad para cada curso académico (2015-2016 y 2016- 2017) bajo la siguiente estructura: 
fundamentación, referentes principales, debilidades, líneas de trabajo metodológico, objetivos generales y específicos, momentos para su aplicación, caracterización del claustro universitario, derivación de las líneas de trabajo metodológico con sus objetivos específicos, el sistema de actividades metodológicas centrales con las precisiones de tema, objetivo, tipo de actividad, ejecutores, fecha y participantes, así como el sistema de control. En las actividades metodológicas centrales participaron especialistas de elevada calificación docente y científica y con incidencia directa en el proceso de formación de pregrado en el eslabón de base. Estas actividades, mediante el efecto de cascada y su contextualización, impactaron muy favorablemente en la mejora de la labor docente metodológica y científico metodológica de la carrera, el año académico, la disciplina y la asignatura; además de posibilitar la sistematización de las mejores experiencias del trabajo metodológico que se desarrolla en las carreras.

El objetivo general que ha guiado la concreción del trabajo metodológico colectivo ha sido:

El perfeccionamiento de la formación integral del profesional mediante la mejora de la gestión didáctica y de dirección en el eslabón de base y el proceso de autoevaluación de las carreras, a fin de garantizar la calidad en el cumplimiento de los objetivos expresados en el modelo del profesional. En el curso académico 2015-2016, materializado en:

- La elevación de los indicadores globales de eficiencia vertical, promoción y promedio de calificación.

- La transformación en los modos de actuación de la comunidad universitaria en el eslabón de base.

- La concreción del enfoque en sistema de los planes de trabajo metodológico y estrategias educativas.

- La motivación y participación estudiantil en la solución de los problemas de su entorno.

En el curso académico 2016-2017, materializado en:

- La elevación de los indicadores globales de eficiencia académica, promoción y promedio de calificación.

- El protagonismo estudiantil en la autogestión del aprendizaje, la participación en la solución de los problemas de su entorno y la satisfacción con su proceso de formación.

- La transformación en los modos de actuación de la comunidad universitaria en el eslabón de base.

- La concreción del enfoque en sistema de los planes de trabajo metodológico.

- La pertinente preparación para los procesos de acreditación de carreras y evaluación institucional.

- La instrumentación de las transformaciones de la educación superior.

- La pertinencia de los ajustes al proceso docente educativo ante contingencias que se deriven de situaciones excepcionales.

Para cumplir con el objetivo general trazado, se definieron líneas metodológicas en los cursos académicos 2015-2016 (vid infra 1,2), 2016-2017 (vid infra 3, 4, 5): 
a. Perfeccionamiento de la gestión didáctica y de dirección desde el funcionamiento de colectivos de carrera, años y disciplinas y la instrumentación de sus estrategias educativas para el mejoramiento de la calidad de la formación del profesional en el eslabón de base.

b. Autoevaluación de carreras como elemento dinamizador de la gestión para la calidad de la formación integral del profesional.

c. Perfeccionamiento de las estrategias educativas y sus resultados en todos los niveles.

d. Perfeccionamiento de la gestión en los niveles organizativos y de dirección del trabajo metodológico, con prioridad en el liderazgo del departamento docente en el eslabón de base.

e. La instrumentación y validación de las transformaciones de la educación superior para el perfeccionamiento de los planes y programas de estudio.

De modo que, desde esas líneas, las prioridades que se jerarquizaron fueron las siguientes:

- El perfeccionamiento de la Estrategia Educativa para la realización de una labor educativa coherente y sistémica desde el nivel de universidad hasta el eslabón de base.

- La implementación de las transformaciones de la educación superior, evaluando parcialmente sus resultados.

- El perfeccionamiento del sistema de trabajo metodológico de la universidad, dinamizado por ajustes al proceso docente ante contingencias, así como por el proceso de autoevaluación de la formación del profesional de pregrado, en estrecha vinculación con la investigación y extensión universitaria, para la elevación de la calidad del proceso formativo, jerarquizando, como nivel de dirección metodológica, el departamento docente.

- El fortalecimiento del vínculo de las carreras con los organismos e instituciones afines con la visión integradora de la práctica laboral, la actividad académica e investigativa en la preparación de los estudiantes.

- El perfeccionamiento del sistema de trabajo que integra la gestión de las facultades y centros universitarios municipales en la planificación, ejecución y evaluación del proceso docente educativo en cada carrera, las diferentes modalidades y planes de estudio.

Como se aprecia, el objetivo general ha sido el mismo, lo cual ha favorecido su validación de uno a otro curso académico, sobre la base de los resultados que se alcanzan en el proceso de formación y los indicadores que permiten su concreción han posibilitado determinar fortalezas y debilidades; en consecuencia, medir la efectividad de su puesta en práctica desde la sistematización de los requerimientos del trabajo metodológico concebido bajo la perspectiva del enfoque comunicativo como mediador.

Este proceso de planificación se articuló desde los procesos de autoevaluación de la calidad de la formación del profesional en carreras, facultades, centros universitarios municipales y la institución (Vicerrectoría Docente, 2017) hasta los procesos de evaluación externa de las carreras y la institución, a cargo de expertos de la Junta de Acreditación Nacional.

\section{Etapa 3. Control, seguimiento y evaluación del proceso docente educativo}


El control y seguimiento al proceso docente educativo se realizó a través de la asesoría sistemática a facultades, centros universitarios municipales, departamentos docentes y carreras y la participación en las visitas integrales a la formación del profesional de pregrado. Estas visitas quedaron incorporadas en el plan de trabajo metodológico de la universidad, se ejecutaron cada mes y en ellas participaron profesores experimentados y vicedecanos docentes de facultades, al concebirse también como espacio que favorece el entrenamiento de esta figura en la conducción del proceso formativo y el seguimiento que se ofrece a los planes de mejora en los procesos de evaluación externa de carreras y de la variable.

3. Formación del profesional de pregrado para la institución. Estas visitas fueron precedidas por un proceso previo de coordinación con los directivos metodológicos de facultades y centro universitarios municipales y tuvieron como objetivo: controlar la calidad y cumplimiento del trabajo metodológico y su incidencia en el proceso formativo del profesional universitario. Su ejecución se acompañó de una guía con acciones que permitieran valorar el cumplimiento del objetivo mediante actividades para su materialización, a saber: la reunión con el claustro del departamento docente, control a actividad metodológica desarrollada preferentemente por el/la jefe/a de departamento, control a clase a profesor con categoría docente inferior (Asistente e Instructor), revisión de evidencias documentales e intercambio con estudiantes con el propósito de valorar el estado de satisfacción con su proceso formativo.

3.1. En la reunión con los departamentos docentes, se indagó sobre diferentes aspectos, entre ellos: el análisis que se realiza sobre los resultados de los principales indicadores de eficiencia del proceso docente educativo, a saber: promoción y eficiencia académica; las acciones realizadas para garantizar un incremento sustancial de la dedicación al estudio por los estudiantes, la atención a los sistemas de evaluación de las asignaturas; las acciones que evidenciaran la prioridad de la labor educativa desde la instrucción; los aseguramientos que garantizaran la comunicación sistemática con los estudiantes para dar respuesta a sus necesidades educativas con efectividad; la atención personalizada a esas necesidades educativas por los profesores guías y tutores, que permitieran verdaderas transformaciones en su crecimiento personal y profesional; el cumplimiento del plan de trabajo metodológico y el de controles a clases; cómo se garantizaba el cumplimiento de los objetivos educativos de cada año en las prácticas laborales; cómo se instrumentaban las estrategias curriculares; el comportamiento del aseguramiento bibliográfico, uso de los laboratorios y plataformas virtuales de aprendizaje; estado de cumplimiento y calidad de la elaboración de los expedientes de las asignaturas. Asimismo, se indagó acerca de la calidad del seguimiento que, desde la sede central, ofrecía la carrera a la formación de sus profesionales que desarrollan el proceso en los centros universitarios municipales.

3.2. La visita a la actividad metodológica (clase metodológica instructiva -CMI) que imparte el jefe del departamento docente se evaluó sobre la base de los indicadores siguientes:

$\checkmark \quad$ Calidad en su concepción y cumplimiento de su estructura.

$\checkmark$ Preparación del docente que la desarrolla. 
$\checkmark$ Pertinencia del problema conceptual metodológico abordado y su concreción a través del tratamiento didáctico en una clase o sistema de clases desde el contenido seleccionado correspondiente a una asignatura del currículo.

$\checkmark$ Rigor en el abordaje de los aspectos científicos y metodológicos.

$\checkmark$ Riqueza y profundidad de la propuesta metodológica para solucionar el problema conceptual metodológico abordado.

$\checkmark \quad$ Calidad del debate entre los docentes.

$\checkmark$ Coherencia entre la propuesta metodológica presentada y los acuerdos proyectados para su instrumentación por los colectivos metodológicos del departamento.

3.3. El control a clase a profesor/a (Asistente o Instructor) del departamento docente, se realizó con el objetivo de evaluar su desarrollo precisando la calidad en su ejecución:

- Categoría y experiencia en el contenido de la clase del controlador.

- Existencia de una guía o modelo de control. Calidad del mismo. Cumplimiento de indicadores expresados en el plan de trabajo metodológico de la universidad.

- Nivel de precisión y argumentación de los señalamientos realizados.

- Tratamiento a los aspectos educativos e instrumentación del principio de educar a través de la instrucción.

- Rigor y justeza de la evaluación emitida en correspondencia con los señalamientos realizados.

- Pertinencia de las recomendaciones realizadas al profesor controlado en correspondencia con los señalamientos realizados.

3.4. La revisión de evidencias documentales, que respaldan el trabajo metodológico desarrollado como vía esencial que contribuye a la calidad de la labor docente en los colectivos metodológicos de los diferentes niveles organizativos del trabajo metodológico colectivo. A partir de las normativas vigentes y los requisitos establecidos, se verifica el cumplimiento de los objetivos de los planes de estudio y para ello se revisa el Plan del Proceso Docente, los programas analíticos de las asignaturas que se imparten en el momento del control y su expediente metodológico, las evidencias de la instrumentación de las estrategias curriculares, el registro de control de asistencia y evaluación de cada asignatura; la estrategia educativa de la carrera la cual toma como punto de partida el modelo del profesional y la del año académico la cual propicia la integración de las clases, el trabajo científico estudiantil y las prácticas laborales con las diferentes tareas de impacto social, deportivas, y culturales, en correspondencia con los objetivos del año académico; los planes de trabajo metodológico y actas de las actividades metodológicas desarrolladas, así como el cumplimiento del sistema de control al proceso docente educativo.

3.5. Intercambio con los estudiantes para constatar los niveles de satisfacción con el proceso formativo en torno a:

Nivel de exigencia y rigor académico del proceso de formación que garantiza un incremento sustancial de la dedicación al estudio, proyectos de investigación en los que participan, atención recibida por parte del profesor principal de año académico, profesor guía y tutor, 
atención a los arrastres y Alumnos Ayudantes, calidad de la práctica investigativa laboral que realizan y atención recibida, satisfacción con las asignaturas del currículo optativo/electivo y el aseguramiento bibliográfico de las asignaturas, utilización real de los materiales docentes existentes en la red, uso de la literatura docente en idioma inglés; utilización real de la computación en las asignaturas, horas que trabajan los laboratorios de computación de uso colectivo, uso de la internet e intranet; comunicación con profesores y dirigentes sobre aspectos relacionados con el proceso de formación.

Los resultados de las acciones ejecutadas durante la visita integral, se expresan en un informe en el que se precisan las fortalezas y debilidades, las cuales son analizadas con los directivos y funcionarios de los niveles correspondientes en facultades, departamentos y centros universitarios municipales. La valoración cualitativa tiene seguimiento a través de las visitas sistemáticas que realizan los asesores de la Dirección de Formación del Profesional, cuya sistematización metodológica contribuye a la retroalimentación sistemática sobre la calidad del proceso docente educativo, a cerrar el ciclo originado con el diagnóstico y a aperturar su continuidad encaminada a la mejora del proceso de formación del profesional a través del trabajo metodológico colectivo en los diferentes niveles organizativos y de dirección y que tiene como escenario principal para el análisis de los resultados que se alcanzan:

- La actividad metodológica central cada mes en la que se desarrollan reuniones metodológicas o clases metodológicas o talleres metodológicos (tipos fundamentales del trabajo docente metodológico) con la participación bimestralmente de los representantes de los niveles organizativos del proceso docente educativo: coordinadores de colectivos de carrera y profesores principales de año académico, y con la participación cada mes de los representantes de los niveles de dirección: vicedecanos docentes de facultades y jefes de departamentos/subdirectores en los centros universitarios municipales. Estas actividades, previstas en el plan de trabajo metodológico de la universidad, permiten la generalización de las mejores experiencias que pueden ser multiplicadas según los contextos formativos.

- La reunión en la primera semana de cada mes con los representantes de los niveles de dirección encargados de la conducción del proceso docente educativo en facultades y centros universitarios municipales y que inciden directamente en el eslabón de base. Este escenario posibilita validar tanto los resultados que se obtienen sistemáticamente en el proceso docente educativo como la instrumentación de los requerimientos para el logro del trabajo metodológico colectivo, mediado por el enfoque comunicativo, con énfasis en las competencias, compromiso y motivaciones profesionales de los participantes, principios éticos y pedagógicos, las reglas básicas de la comunicación y las fortalezas y debilidades en el tránsito por las etapas definidas para la materialización del trabajo metodológico colectivo.

- Constatar con objetividad los resultados integrales del proceso de formación de pregrado al concluir cada semestre del curso académico.

Todo ello ha posibilitado actualizar los informes de autoevaluación y planes de mejora, con lo cual ha sido posible evaluar sistemáticamente la calidad de la formación, considerando la articulación dialéctica entre resultados y efectividad de la gestión que lo asegura y que tiene su expresión en la sostenibilidad de las fortalezas, la determinación de debilidades y actualización de los planes de mejora a todos los niveles, constituyendo el proceso descrito 
en su conjunto una experiencia renovadora que ha sido validada durante dos cursos académicos en la Universidad de Oriente. Los principales impactos como resultado de la sistematización que se valida se sintetizan en las fortalezas que se presentan a continuación:

- Tratamiento más efectivo y de mayor calidad a los problemas metodológicos y líneas que se atienden en cada uno de los niveles organizativos y de dirección, como resultado de una concepción integral, coherente y pertinente del trabajo metodológico colectivo en facultades y centros universitarios municipales y que tiene su expresión en un incremento tendencial de los principales indicadores del proceso de formación de pregrado: promoción y eficiencia académica vertical y limpia.

- Mejor gestión de los colectivos metodológicos que ha favorecido la calidad del proceso formativo, y que se revierte en la elevada satisfacción de los estudiantes con la calidad de la formación del profesional a partir del reconocimiento al desempeño del claustro en su labor docente metodológica y educativa, con la puesta en práctica de métodos y medios que constituyen alternativas para el logro de los objetivos del modelo del profesional.

- Mayor impacto de las actividades metodológicas realizadas, con una concepción más pertinente de los planes elaborados, tanto en la cantidad general como en su diversidad según las diferentes tipologías, las que han contribuido a elevar la calidad del proceso docente educativo.

- Fortalecimiento del trabajo científico metodológico con la realización de los seminarios en departamentos docentes, facultades y centros universitarios municipales y la organización de la Conferencia científico metodológica a nivel de universidad (CONCIMET 2016 y 2017) para generar, introducir y divulgar los resultados de investigaciones y experiencias pedagógicas.

- Prioridad al trabajo metodológico dirigido a la preparación de la asignatura, lo que se materializó en la elevación de la calidad de sus expedientes metodológicos.

- Mayor pertinencia del trabajo metodológico colectivo en el seguimiento a debilidades identificadas mediante los procesos de autoevaluación de carreras e institución y que le permiten a la Universidad de Oriente exhibir 15 carreras de excelencia, 16 certificadas, 2 avaladas, de las 37 acreditables en el curso diurno, para un 89,2 \% de carreras con una categoría superior de acreditación. Asimismo, haber transitado exitosamente por el proceso de evaluación externa institucional con el reconocimiento expresado en el informe elaborado por expertos de la Junta de Acreditación Nacional, lo cuales se harán públicos en 2018.

\section{CONCLUSIÓN}

La validación de la experiencia relativa a la implementación del sistema de trabajo metodológico en la Universidad de Oriente, se sustenta en el trabajo metodológico colectivo mediado por un enfoque comunicativo como premisa para su desarrollo, el cual deviene en elemento esencial de la gestión metodológica realizada en el contexto de la integración de la actual Universidad de Oriente. 
La concepción integral bajo la cual fue diseñado, organizado, planificado, controlado y evaluado el trabajo metodológico colectivo considerando el papel que desempeña la comunicación para su concreción, permitió incidir de manera positiva en la labor metodológica del claustro que se concreta en el eslabón de base de la comunidad universitaria del año académico.

El trabajo metodológico colectivo se ha desarrollado bajo una concepción integral y sistémica que ha posibilitado alcanzar transformaciones en el eslabón de base y en los diferentes niveles organizativos y de dirección de la universidad en general y el proceso docente educativo en particular, mediante una gestión más pertinente y efectiva que impacta positivamente en la formación integral del profesional. Esas transformaciones se sintetizan en fortalezas, que son expresión de la sostenibilidad de la calidad del proceso formativo en la Universidad de Oriente.

\section{BIBLIOGRAFÍA}

1. Abello Cruz, Ana María et al (2013). El mundo y la cultura mediados por la lengua. La Habana, Editorial Pueblo y Educación.

2. Cordoves - Mustelier, Dicsiel y Bertha Fernández (2014). La gestión universitaria del trabajo metodológico aplicando cuatro instrumentos en cinco universidades cubanas. La Habana, Instituto Superior Politécnico José Antonio Echeverría, ISSN 18155936/Vol. XXXIV/No. 2/septiembre- diciembre/2014/ p. 289-300. Recuperado de http://scielo.sld.cu/pdf/rii/v35n3/rii05314.pdf.

3. Domínguez, Ileana (2007). Comunicación y texto. La Habana, Editorial Pueblo y Educación.

4. Fonseca Arias, B. et al (2016). El trabajo metodológico colectivo. Una experiencia en perfeccionamiento desde la Dirección de Formación del Profesional en la Universidad de Oriente. Sello Editor MINED, ISBN 978-959-18-1137-0.

5. Horruitiner S., Pedro (2009) La universidad cubana: el modelo de formación. La Habana, Editorial Universitaria, ISBN 978-959-16-0676-1.

6. Howard, Gardner (2008). Inteligencias múltiples.La teoría en la práctica. Paidós. ISBN 978-607-7626-00-8. Recuperado de http://ict.edu.ar/renovacion/wpcontent/uploads/2012/02/Gardner_inteligencias.pdf.

7. Junta de Acreditación Nacional (2015). Sistema de Evaluación y Acreditación de Instituciones de Educación Superior (SEA-IES). La Habana, Editorial Universitaria Félix Varela.

8. Ministerio de Educación Superior (2007). Reglamento de Trabajo Docente y Metodológico, Resolución No. 210/2007. Impreso en la UEB de producciones gráficas, septiembre.

9.

(2013). Perfeccionamiento del sistema de gestión del proceso de formación integral de los estudiantes universitarios en el eslabón de base. Editorial Universitaria Félix Varela.

10. Vicerrectoría Docente (2017). Informe de autoevaluación de la variable 3: Formación del Profesional de Pregrado. Universidad de Oriente, Santiago de Cuba. 